\title{
Complicated workpiece shape machining using optimal target shape position and orientation on a multifunctional machine tool
}

\author{
Maho KUMANOTANI* and Keiichi NAKAMOTO* \\ *Tokyo University of Agriculture and Technology, \\ 2-24-16 Naka-cho, Koganei, Tokyo 184-8588, Japan \\ E-mail: kuma.tuatm12@gmail.com
}

Received: 20 December 2017; Revised: 11 April 2018; Accepted: 14 June 2018

\begin{abstract}
In order to machine complicated workpiece shapes, it is essential for operators to avoid detrimental collisions between machine structures and workpieces. Therefore, a machine simulator based on 3D models is usually utilized prior to machining operations to ensure this problem is avoided. On the other hand, a multifunctional machine tool with both machining and additive manufacturing (AM) functions is recently developed to realize high flexibility and productivity. However, it is difficult to predict and control workpiece shapes created by AM. Such shapes are generally different from the 3D model prepared in advance. The differences may happen unexpected collisions on a multifunctional machine tool. Additionally, machining productivity may decrease when a $\mathrm{NC}$ program is generated with an original $3 \mathrm{D}$ model that is different from the actual workpiece shape. Thus, this study aims to realize highly efficient machining of complicated workpiece shapes corresponding to actual workpiece shapes as created by AM. In order to eliminate differences between the 3D model and the actual workpiece shape, a 3D model is firstly created by measuring the shape and position of the workpiece on a multi-functional machine tool. Then, appropriate position and orientation of the target shape is decided to minimize tool path distance. From results of a case study, it is appeared that the proposed machining procedure can improve machining efficiency of complicated workpieces as created by AM.
\end{abstract}

Keywords : Complicated workpiece shape, CAM, Multifunctional machine tool, On-machine measurement, Additive manufacturing, Structure from motion

\section{Introduction}

In the area of machining, it is well known that complex machine tool motions may cause unexpected collisions between a workpiece and machine tool elements including a jig. Such collisions can damage or even destroy the workpiece, cutting tool or machine tool (Abele and Korff, 2011). Therefore, in order to realize highly efficient machining, it is necessary to use a machine simulator prior to machining operations to detect and avoid potential collisions. On the other hand, the manufacturing industry switched to high-mix low-volume production, and it is also necessary to cut production lead-time and costs. Thus, multifunctional machine tools that allow the integration of traditional machining and other manufacturing operations such as additive manufacturing (AM) have recently been developed to satisfy these demands.

However, it is difficult to predict and control workpiece shapes created by AM correctly, and collisions can occur on a multifunctional machine tool due to differences between the 3D model prepared in advance and the actual object. Although several previous studies have investigated collision detection and avoidance (Lacharnay, et al., 2015; Watanabe, et al., 2017) by modifying tool trajectories and using machine simulation, they have not considered the differences of workpiece shape that may induce collisions. Additionally, machining efficiency may decrease when NC program is generated based on a 3D model different from the actual object.

Thus, this study aims to realize highly efficient machining of complicated workpiece shapes corresponding to an actual workpiece shape as created by AM. This study utilizes a 3D model created based on on-machine measurement by 
structure from motion (SfM), which is a technique to estimate a 3D shape from a series of 2D images (Baier and Rando, 2016). Then, the position and orientation of the target shape can be set to shorten tool path distance based on the $3 \mathrm{D}$ model of the actual workpiece shape created by on-machine measurement. The effectiveness of the proposed machine procedure is verified by conducting a case study assuming complicated workpiece shapes as created by AM on a multifunctional machine tool.

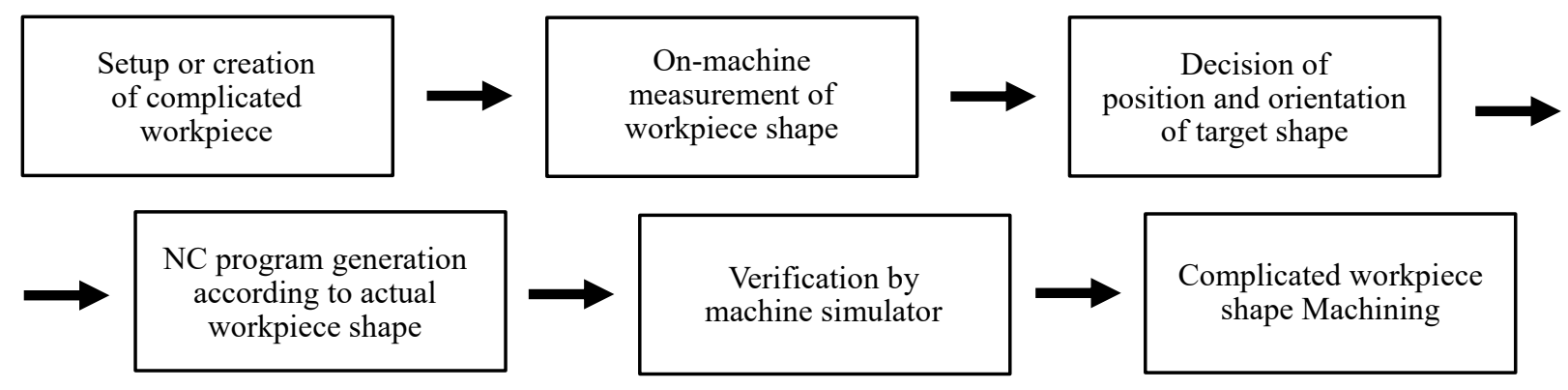

Fig. 1 Flow of proposed machining procedure.

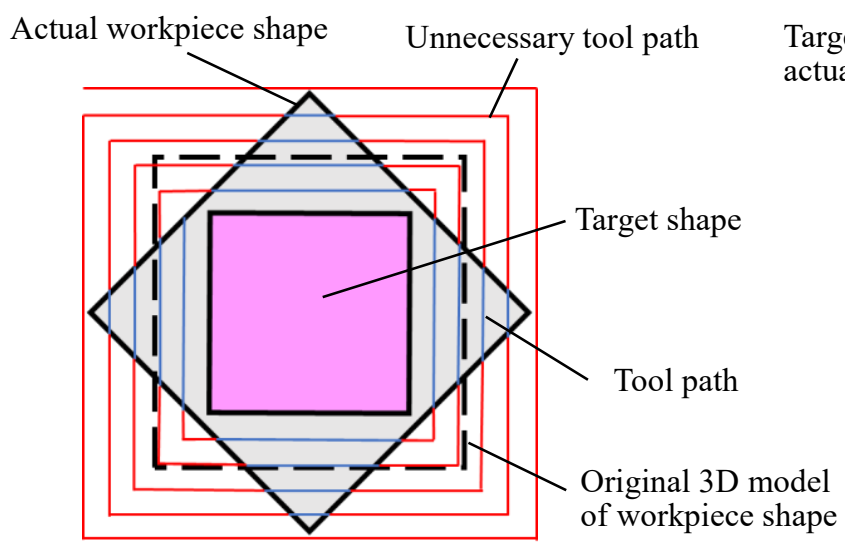

(a) Tool path based on original workpiece shape.

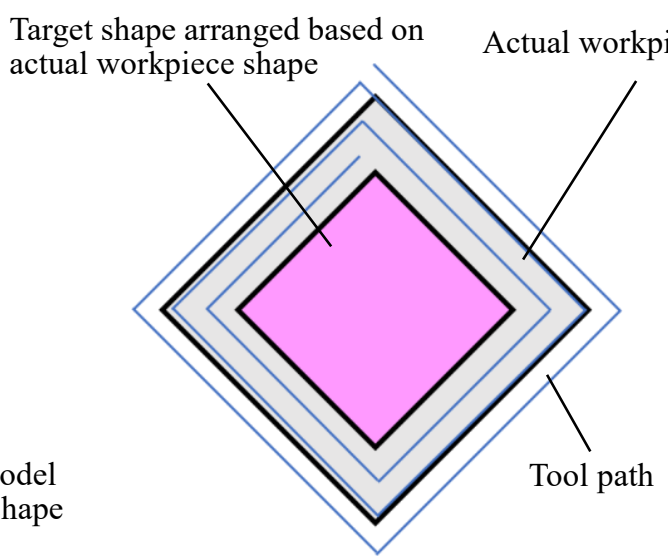

(b) Tool path based on actual workpiece shape.

Fig. 2 Comparison of tool path generated based on workpiece shape.

\section{Tool path generation for collision-free, highly efficient machining}

In a conventional machining procedure, it is essential for operators to avoid damaging collisions between machine structures and a workpiece. As a result, a lot of researches on the avoidance of collisions between a workpiece and machine tool elements have been actively conducted, especially in multi-axis control machining. Kanda and Morishige proposed a tool path generation method considering collisions among cutting tool, workpiece, jig and machine structures by 2D C-Space (Kanda and Morishige, 2012). They reduced processing time using a graphics-processing unit (GPU) and polygon models. Additionally, they applied the method to all types of 5-axis control machine tools based on coordinates transformation. Hu et al. addressed the obstacle avoidance problem by considering non-static obstacles as fixtures and clamps on the machine table and devices fixed in the machine space (Hu, et al., 2013). They classified obstacles into three types: part of the workpiece (including the surface or in-process geometry model), things attached to or fixed on the machine table and things attached to or fixed on the machine's base. They then expressed all types of obstacles mathematically. Chen et al. proposed a reference plane-based algorithm for avoidance of collisions and generation of a set of smoothly aligned tool orientations for the machining of a blade disk (Chen, et al., 2015). However, these studies assumed that a workpiece shape can be known correctly in advance. Thus, this study deals with collision avoidance between a workpiece and machine tool elements that may occur when the workpiece shape is different from the 3D model created in advance.

In this study, a novel machining procedure is proposed, as shown in Fig. 1. In order to eliminate differences between 
a 3D model and actual workpiece shape, the 3D model is created by measuring the shape and position of the workpiece after setup or creation on a multifunctional machine tool. The machining efficiency of a complicated workpiece is strongly affected by the position and orientation of a target shape. However, there are few researches about the decision method of target shape position and orientation based on the workpiece shape. Although Ogawa, et al. proposed a method corresponding to a non-uniformly shaped workpiece, they have not considered complicated 3D workpiece shapes (Ogawa, et al., 2012). Therefore, in this study, the decision method of appropriate position and orientation of the target shape is proposed, based on the complicated workpiece model obtained by on-machine measurement. Then, the NC program is generated according to the obtained workpiece shape and appropriately positioned target shape. After verification by a machine simulator, complicated workpiece shape machining is finally conducted.

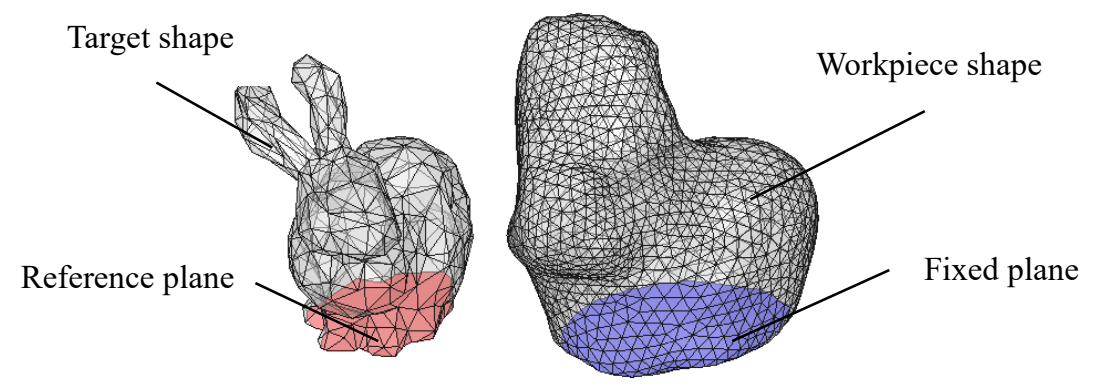

Fig. 3 Fixed plane of workpiece shape and reference plane of target shape.

The position and orientation of the target shape are decided to improve the machine efficiency of rough machining with contour-line path in this study. When the target shape is located at an inappropriate position, rough machining efficiency may decrease due to the long tool path generated in correspondence to an original 3D model of the target shape. For example, unnecessary tool paths often occur as shown in Fig. 2. Therefore, there is an effort to locate the target shape around the center of the workpiece shape with appropriate orientation. In order to demonstrate the decision method, the bunny target shape depicted in Fig. 3 is used in this study. 3D models of target shape and workpiece shape are treated based on STL format because the data format is the de facto standard in the area of AM. It is assumed that a workpiece shape contains the target shape, and that the workpiece shape and target shape have a fixed plane and a reference plane, respectively. These planes are positioned on the same plane when attached to the machine tool table. Additionally, the position and orientation of the target shape are determined only by translation on the XY plane and rotation around the axis parallel to $\mathrm{Z}$ axis in the machine coordinate system. As a result, the position of the target shape is not changed in the height $(Z)$ direction.

\section{Proposed machining procedure \\ 3.1 Creation of 3D model by on-machine measurement}

On-machine measurement has been utilized for various objects in the area of machining, such as workpiece shape (Chen, et al., 2010), tool wear (Chen, et al., 2016) and machine tool accuracy (Szipka, et al., 2018). In the proposed machining procedure, a 3D model is first created based on on-machine measurement. Touch-trigger probes are well adopted as the method of on-machine measurement because of good communication capability with a NC system (Ibaraki, et al., 2013). However, touch-trigger probes are unsuitable for measuring wide area in the machining space. Therefore, non-contact measurement by SfM is utilized in the proposed machining procedure. SfM is a technique for reconstructing the 3D shape of an object from a series of 2D images. This technique is usually adapted in archaeological investigation and does not require special equipment like laser measurement (Ukita and Takahiro, 2016) and calibration of utilized camera is not required as general photo-measurement (Luhmann, et al., 2016). SfM consists of three main steps: extracting image features; matching features among 2D images; and estimating 3D shapes. Image features do not rely on the position of shots taken and allow a single feature to be correctly matched with high probability (Lowe, 2004), such as scale invariant feature transform (Dou and Li, 2013; Hossein-Nejad and Nasri, 2017). 


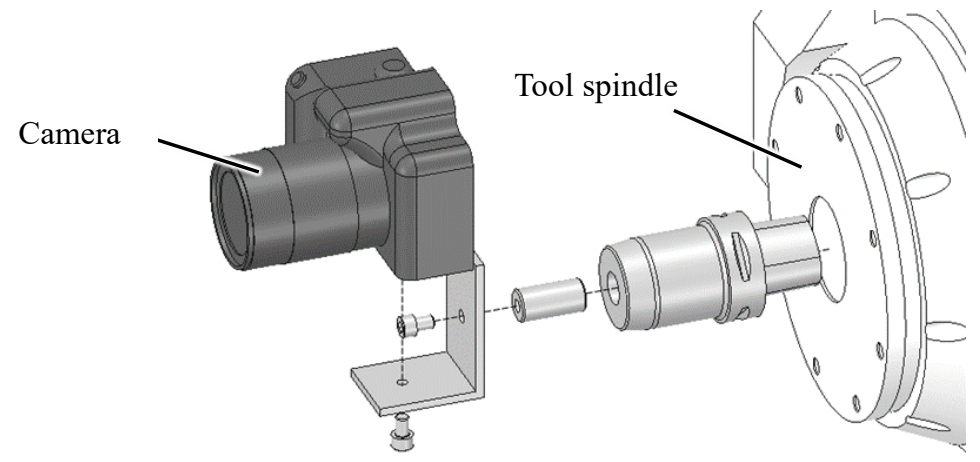

Fig. 4 Single-lens reflex camera attached to tool spindle.

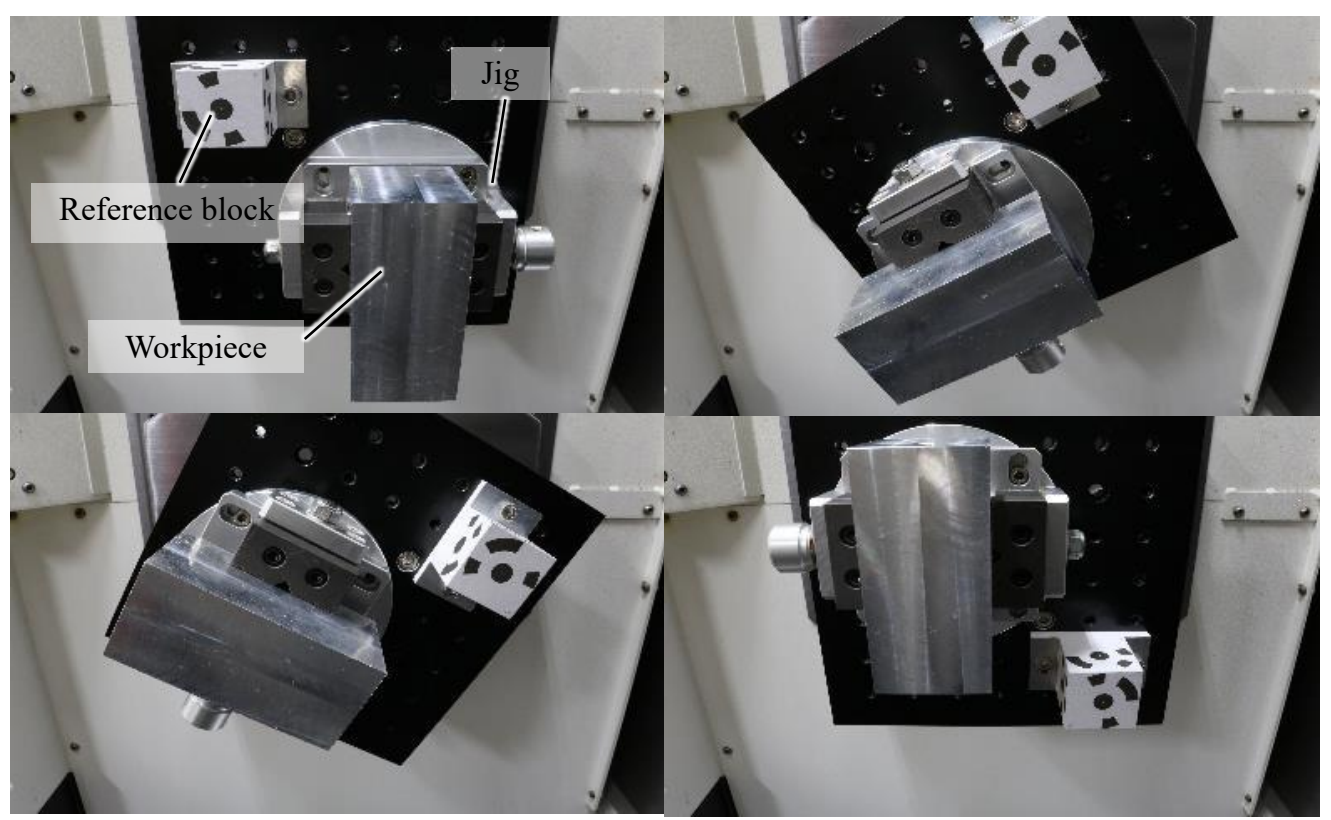

Fig. 5 A series of 2D images of workpiece, jig and reference block for SfM.

Table 1 Camera specifications and image acquisition conditions.

\begin{tabular}{|l|l|}
\hline Diaphragm & F16 \\
\hline Sensitivity & ISO 800 \\
\hline Shutter speed s & 0 '5 \\
\hline Number of shots (per angle) & 60 \\
\hline Camera angle deg. & $45,55,70$ \\
\hline Effective pixels $\times 10^{4}$ pixel & 2420 \\
\hline
\end{tabular}

In this study, in order to use this technique on a multifunctional machine tool, a single-lens reflex camera is attached to the tool spindle via a jig, as shown in Fig. 4. A reference block of known size was fixed near a workpiece to detect its size and position on a machine tool table. 2D images of the workpiece shape are obtained with the camera attached to the tool spindle by rotating the machine tool table at regular intervals.

In order to confirm the feasibility of on-machine measurement by SfM, a square workpiece model, including jig and reference block, are created. Figure 5 shows 2D images of obstacles obtained using the attached camera. Table 1 summarizes the specification of the camera (Canon EOS kiss X8i) and image acquisition conditions. 


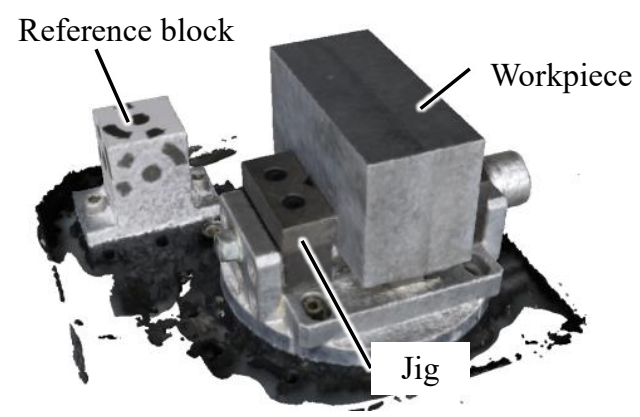

Fig. 6 3D models of workpiece, jig and reference block created by SfM.

A 3D model is created based on obtained images using commercial software (Agisoft Photoscan Professional 1.2.4). The image acquisition conditions are determined based on the recommended circumstance the SfM software. For example, the software requires that at least $60 \%$ of adjacent photographs are overlapped each other. Thus, the number of shot and the camera angle are determined to meet this requirement. Additionally, the images are obtained without using a flash. Figure 6 shows the 3D models created by SfM. A comparison of the results with CMM images (Mitsutoyo CrystaPlus M776) indicates that 3D models can be created within several hundreds $\mu \mathrm{m}$ errors, even with on-machine measurement.

\subsection{Set of initial position and orientation of target shape}

As mentioned in section 2, a target shape should be located around the center of a workpiece shape to shorten tool path distance. Therefore, a decision method for obtaining the appropriate position and orientation of a target shape is devised in this study. In order to accelerate the arrangement of the target shape, the position and orientation are roughly decided at the first step. The decision method is divided into two steps: (1) setting up the initial position and orientation; and (2) changing it to the finial position and orientation.

The initial position and orientation of a target shape are set using a point matching algorithm between two points. The matching algorithm is applied to two point sets, $\boldsymbol{X}=\left\{\boldsymbol{x}_{\boldsymbol{i}}\right\}_{\boldsymbol{i}=\mathbf{1}}^{\boldsymbol{n}}$ and $\boldsymbol{Y}=\left\{\boldsymbol{y}_{\boldsymbol{i}}\right\}_{\boldsymbol{i}=\mathbf{1}}^{\boldsymbol{n}} \subset \mathbb{R}^{\mathbf{3}}$, which are written:

$$
y_{i}=R x_{i}+T+N_{i}
$$

where $\boldsymbol{R}$ is a $3 \times 3$ rotation matrix, $\boldsymbol{T}$ is a translation vector and $\boldsymbol{N}_{\boldsymbol{i}}$ is a noise vector. The point matching algorithm estimates $\boldsymbol{R}$ and $\boldsymbol{T}$, which minimize noise vectors. In other words, the following function is minimized as:

$$
\Sigma^{2}=\sum_{i=1}^{N}\left\|y_{i}-\left(R x_{i}+T\right)\right\|^{2}
$$

Several methods for estimating $\boldsymbol{R}$ and $\boldsymbol{T}$ have been proposed (Horn, 1987; Horn, et al., 1988; Walker, et al., 1991). This study adopts the point matching algorithm based on the singular value decomposition (SVD) (Arun, et al., 1987), because the algorithm is simple and easily implemented.

Divided target shape segment

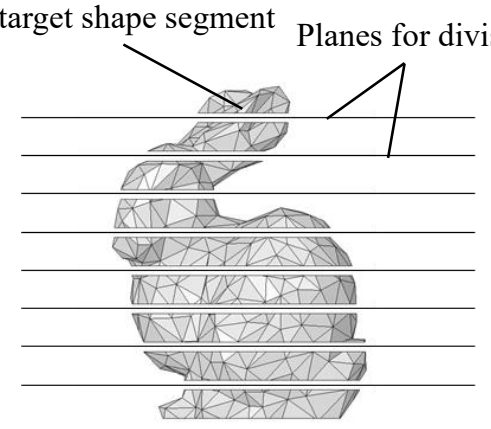

Fig. 7 Division of a model at regular intervals.

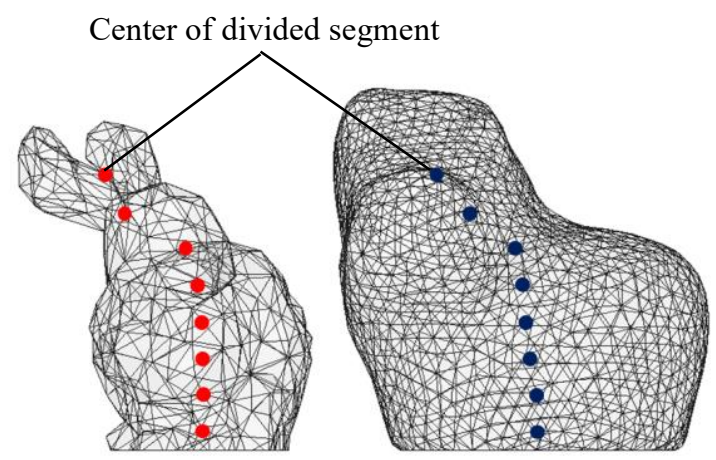

Fig. 8 Center points created for initial position and orientation. 


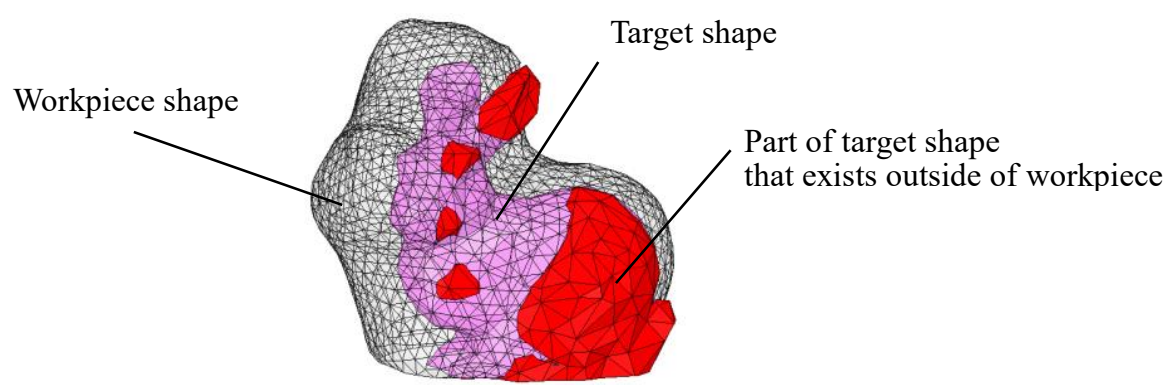

Fig. 9 Set of initial position and orientation.

In order to create two point sets for point matching, both a target shape and a workpiece shape are divided by planes at regular intervals. The planes are parallel to the XY plane, as shown in Fig. 7, and the center points of each divided segment are obtained, as shown in Fig. 8.

The center points in the same $\mathrm{Z}$ coordinate are then defined as corresponding points. The matching algorithm based on SVD is applied to the point sets of center points. At this step, the target shape might not to be included within the workpiece shape, as shown in Fig. 9.

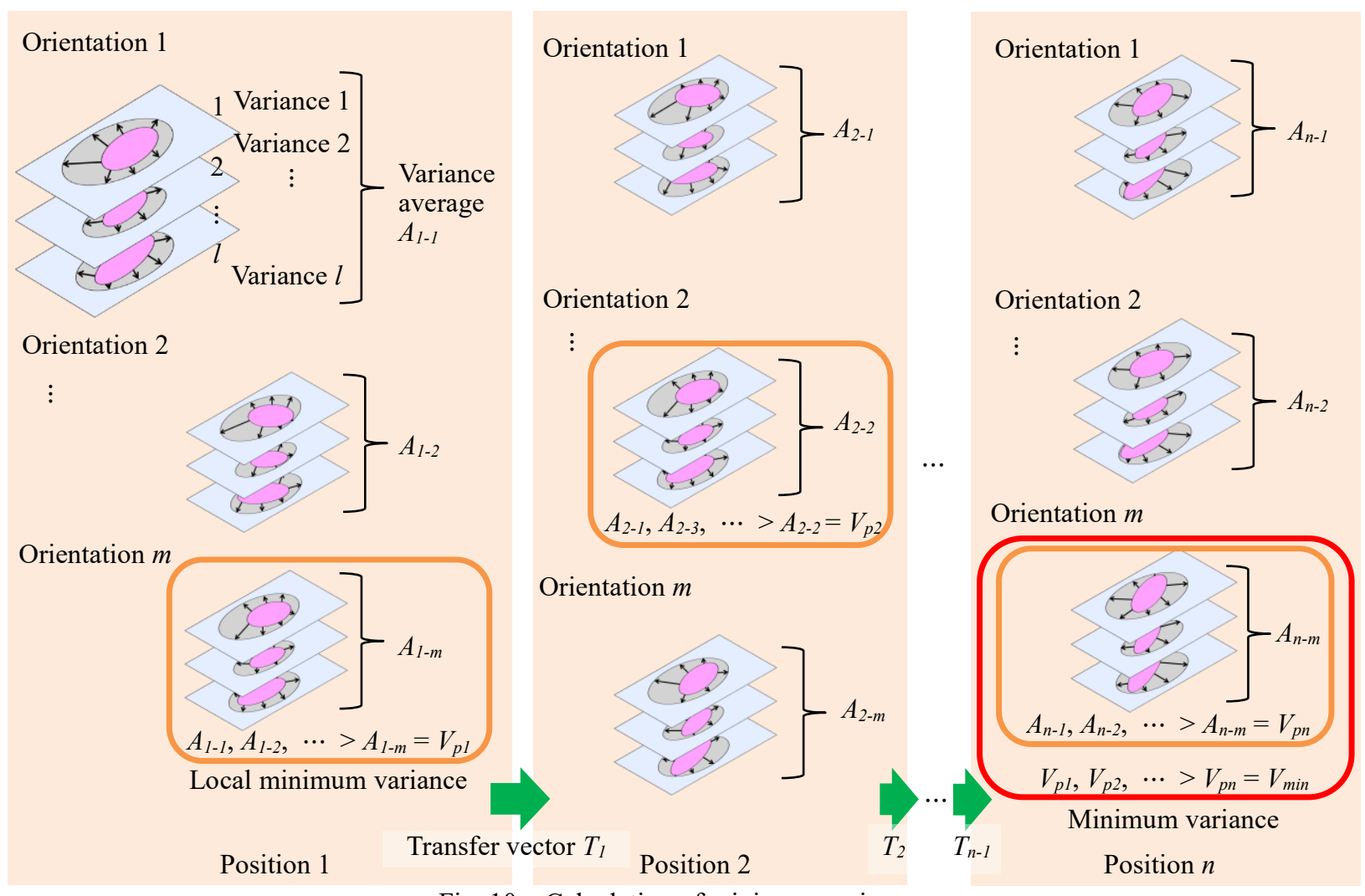

Fig. 10 Calculation of minimum variance.

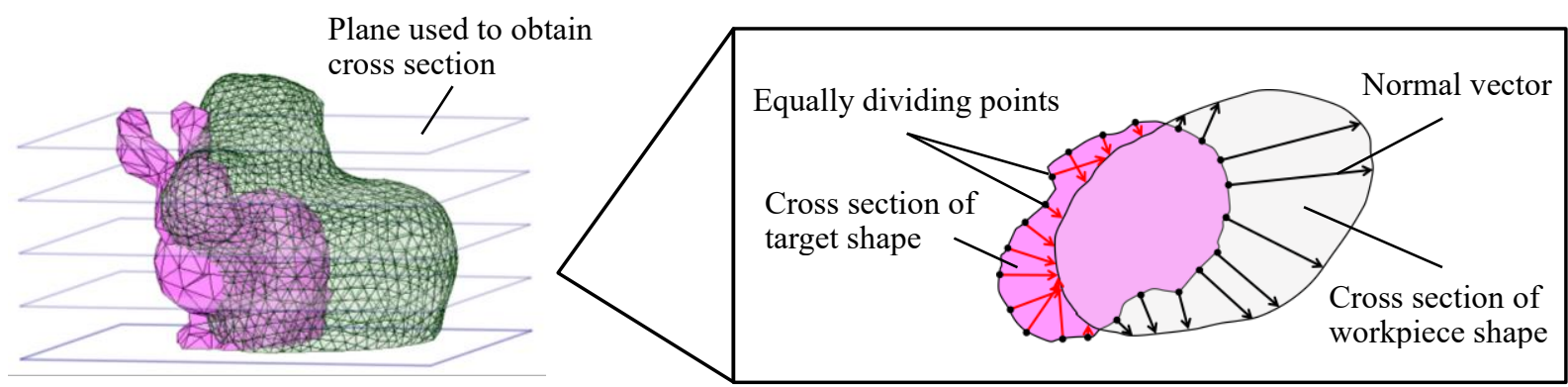

Fig. 11 Normal vector and points equally dividing cross section profile of target shape. 


\subsection{Change to final position and orientation of target shape}

Final position and orientation of the target shape are obtained using normal vectors from the target shape surface and the variance of normal vector lengths. When a target shape is located around the center of a workpiece shape, the normal vectors, which are parallel to the XY plane and exist in the same $\mathrm{Z}$ coordinate, have almost the same length and the variance of the normal vector lengths is close to zero. Therefore, the normal vectors parallel to the XY plane are generated at regular $Z$ coordinate intervals and the variances of normal vector lengths are calculated for each $Z$ coordinate. The average of calculated variances is called the variance average and used to decide the target shape position and orientation. In this study, the target position and orientation are defined where the variance average have the smallest value as the target shape position and orientation are changed. As shown in Fig. 10, the smallest value of variance average $V p_{j}(j=$ $1,2, \cdots)$ was sought for each target position after the variance average $A_{j-m}$ is obtained at each orientation. The final target shape position and orientation are set where the variance average had the smallest value $V_{\min }$. A more detailed description of this method is presented below.

Cross sections of a target shape, parallel to the XY plane, are prepared first. These cross sections do not always match the planes used to set the initial position and orientation, and normal vectors existing on the same plane are created in each cross section as shown in Fig. 11. The normal vectors start from points equally dividing the profiles of cross sections and reach to a workpiece shape surface. If the start points exist inside the workpiece shape, the normal vectors turn toward the outside of the target shape. Otherwise, the normal vectors turn toward the inside of the target shape. When the entire target shape exists inside the workpiece at the initial position and orientation, the variance average is calculated as the initial value of $V_{\min }$.

Vectors called transfer vectors change the target shape position. A transfer vector is calculated for each $\mathrm{Z}$ coordinate based on normal vectors that exist in the same $Z$ coordinate. In order to calculate the transfer vectors, the averages of $\pm X$ and $\mathrm{Y}$ components of normal vectors $\left(\bar{x}_{+}, \bar{x}_{-}, \bar{y}_{+}\right.$and $\left.\bar{y}_{-}\right)$are calculated in each $\mathrm{Z}$ coordinate. The $\mathrm{X}$ and $\mathrm{Y}$ components of the transfer vector are calculated as $\left(\bar{x}_{+}+\bar{x}_{-}, \bar{y}_{+}+\bar{y}_{-}\right)$respectively. The final transfer vector used to arrange the target shape is the average of transfer vectors of whole cross sections.

After the target shape position is determined based on the transfer vector, the target shape orientation is changed by rotating the target shape around the center of a rectangle that includes the target shape and the local minimum variance average is obtained as $V_{p j}$. By comparing the local minimum variance averages of all the target positions, the final position and orientation is set where the minimum variance $V_{\min }$ is obtained.

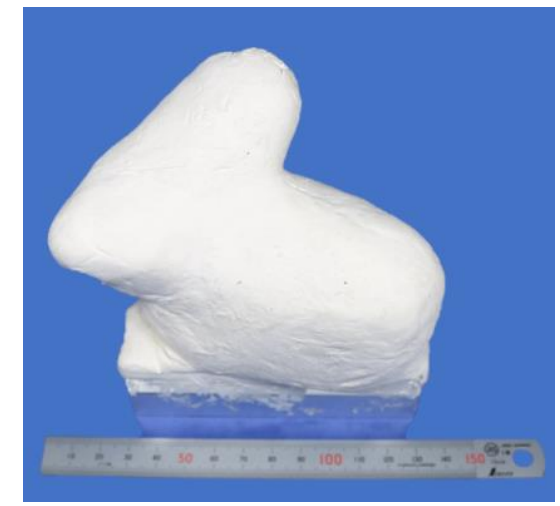

Fig. 12 Workpiece for case study.

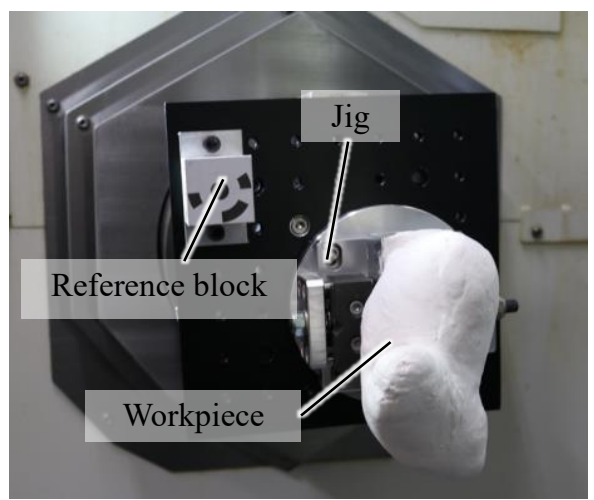

Fig. 13 Setup of workpiece and reference block on machine tool table.

\section{Case study}

In order to verify the decision method of target position and orientation in the proposed machine procedure, a case study using the workpiece created by on-machine measurement is conducted. The machining efficiency is compared with different positions and orientations of a target shape. The original target shape position and orientation assumed before setup are adopted in Case A. In Case B, the initial target position and orientation decided roughly by the proposed method are adopted based on the created workpiece model by SfM. In Case C, the final target shape position and orientation 
decided by the proposed method are adopted. In this case study, the target shape of a bunny is used, and a workpiece made from clay is prepared to simulate a complicated shape as created by AM, as shown in Fig. 12.

$3 \mathrm{D}$ model of the workpiece is created first by on-machine measurement using SfM. Figure 13 shows the setup of the workpiece and reference block on a machine tool table. After the setup, the position of the reference block on the machine tool is measured with a touch-trigger probe installed on a tool spindle.

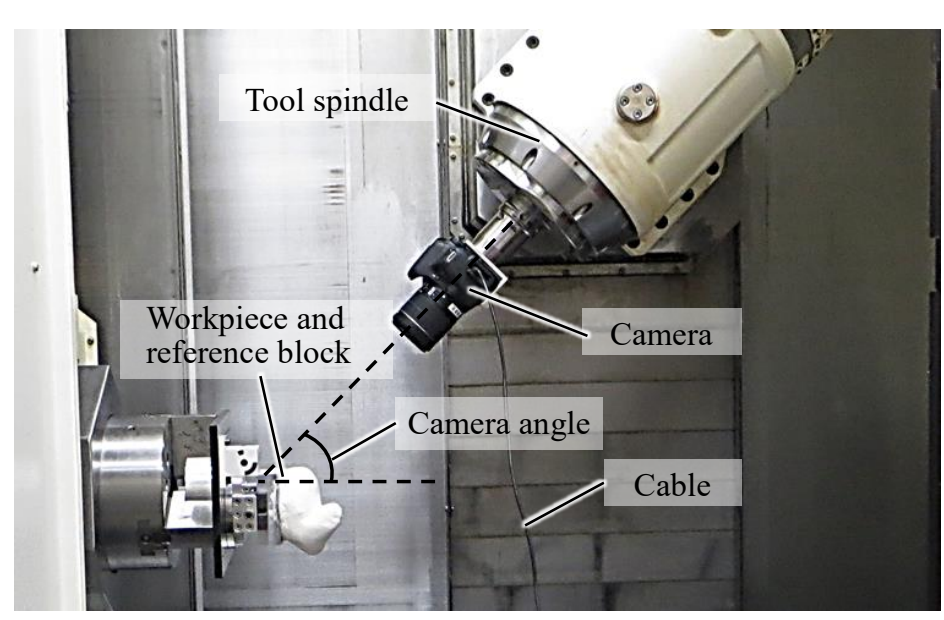

Fig. 14 Overview of image acquisition for SfM on machine tool.

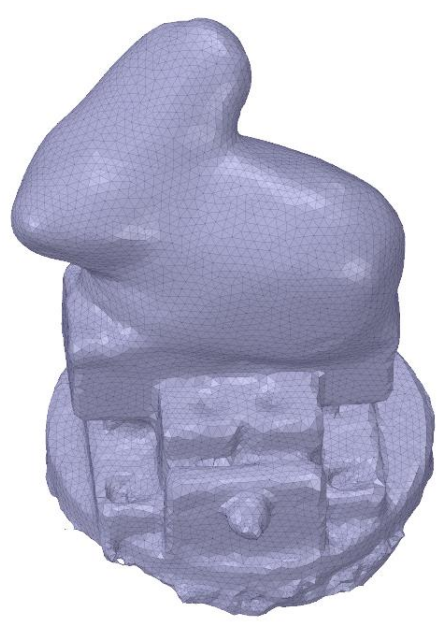

Fig. 15 3D model of workpiece created by SfM.

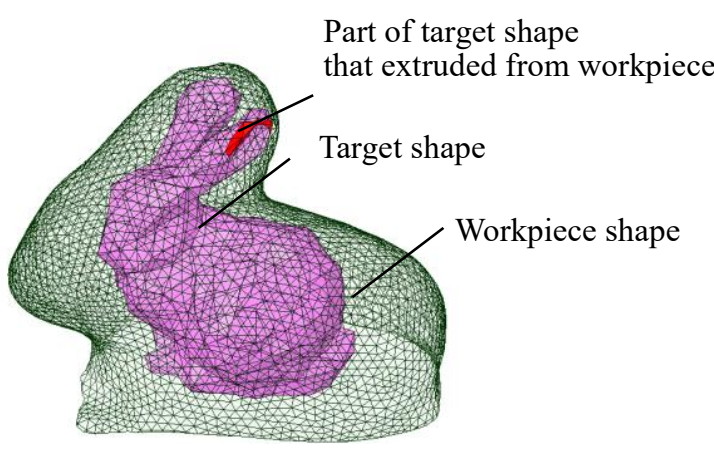

(a) Position and orientation decided by the center of gravity.

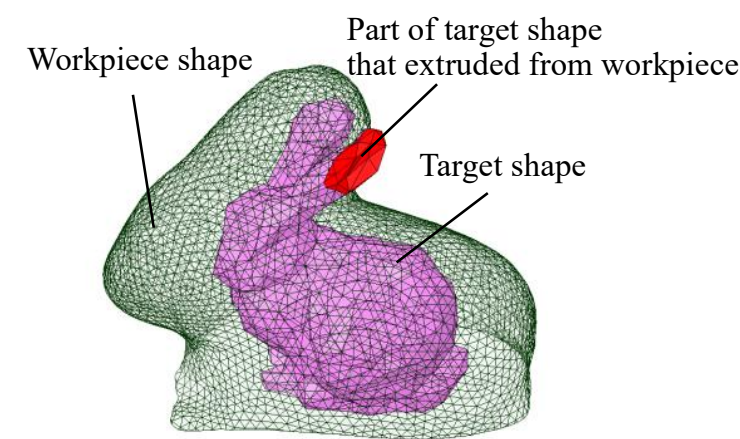

(b) Position and orientation decided by the centers of reference and fixed plane.

Fig. 16 Irrelevant target shape positions and orientations for complicated workpiece shape machining.

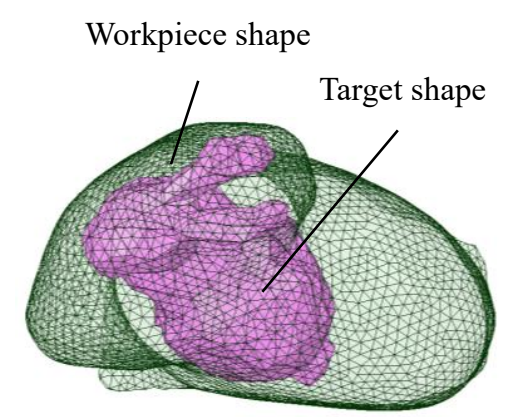

(a) Original position and orientation.

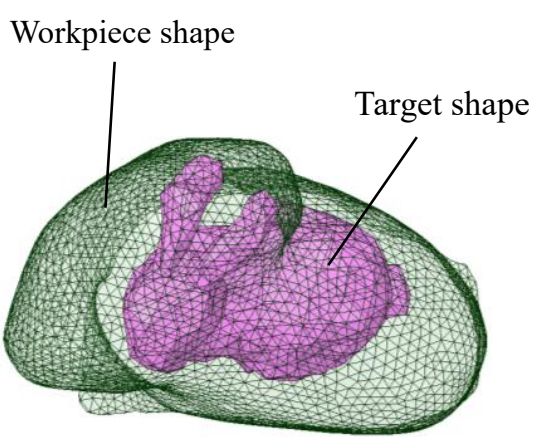

(b) Initial position and orientation.

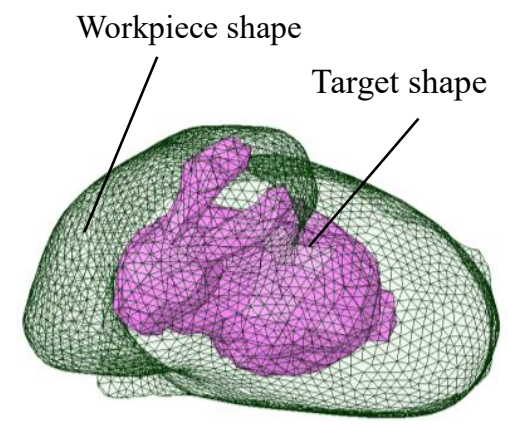

(c) Position and orientation decided by proposed method.

Fig. 17 The final target shape position decided for the case study.

A series of photographs for SfM are obtained from the setup shown in Fig. 14. The image acquisition conditions are changed according to the shape and size of the targeted workpiece as following. The camera angles of the single lens 
reflex camera are 15, 30, 45, 50 and $60 \mathrm{deg}$., with 60 shots taken per angle. The camera is connected to a computer by a cable and controlled remotely. The 3D model of the workpiece is created using Agisoft software (Photoscan Professional 1.2.4), as shown Fig. 15.

The position and orientation of the target shape are decided based on the created 3D model of the workpiece. In this case study, 24 center points are used to set the initial target position and orientation, the interval for obtaining cross sections for generating the normal vectors is $3 \mathrm{~mm}$ and there are 30 normal vectors on the same plane. For example, two other cases are prepared by matching both the centers of gravity of the target and workpiece shapes and the centers of reference and fixed planes. In these cases, the target shape is not included inside the workpiece in any orientation, as shown Fig. 16. From the above, it may be easily understood that the target shape position and orientation cannot be decided for complicated workpiece shapes.

Figure 17(a) shows the original target shape position and orientation assumed in advance; (b) shows the initial target shape position and orientation roughly decided by proposed method; and (c) shows the final target shape position and orientation by the proposed method. In this case study, in order to compare machining efficiencies, tool paths are generated according to each target shape position and orientation.

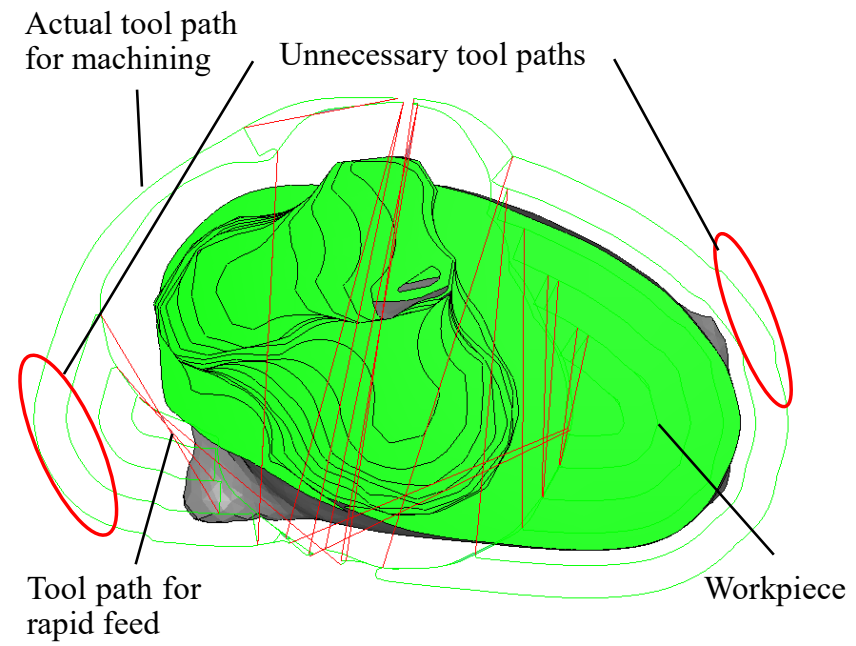

(a) Tool path based on the original target shape position and orientation.

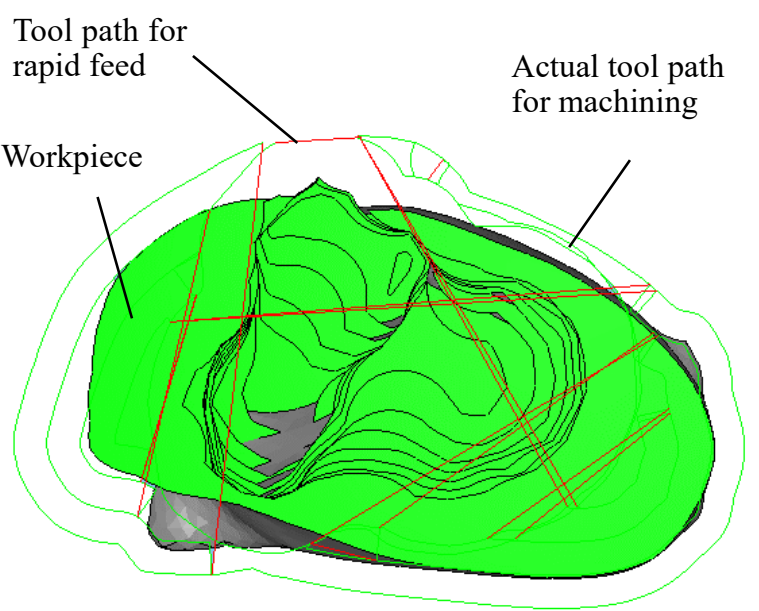

(b) Tool path based on the initial target shape position and orientation.

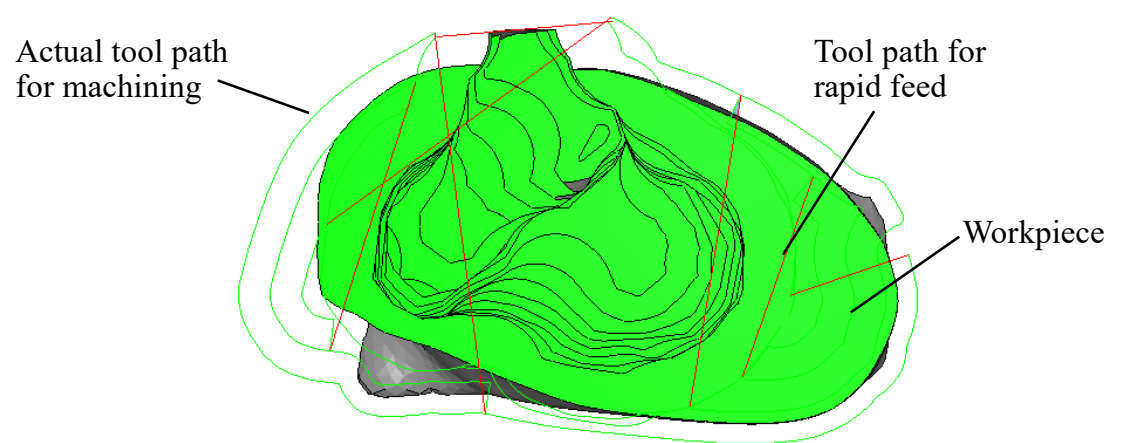

(c) Tool path based on the target shape position and orientation decided by the proposed method.

Fig. 18 Differences between generated tool paths according to target shape position and orientation. 
Table 2 Comparison of results of machining efficiency according to target shape position and orientation.

\begin{tabular}{|c|c|c|c|}
\hline & $\begin{array}{c}\text { Case A } \\
\text { (Original position and orientation) }\end{array}$ & $\begin{array}{c}\text { Case B } \\
\text { (Initial position and orientation) }\end{array}$ & $\begin{array}{c}\text { Case C } \\
\text { (Final position and orientation) }\end{array}$ \\
\hline $\begin{array}{l}\text { Machining time } \\
\text { min }\end{array}$ & 39.8 & 35.4 & 29.8 \\
\hline $\begin{array}{l}\text { Tool path distance } \\
\mathrm{m}\end{array}$ & 29.7 & 26.3 & 22.2 \\
\hline $\begin{array}{c}\text { Removal volume } \\
10^{5} \mathrm{~mm}^{3}\end{array}$ & 1.72 & 1.65 & 1.62 \\
\hline $\begin{array}{l}\text { Removal volume per } \\
\text { tool path distance } \\
10^{3} \mathrm{~mm}^{3} / \mathrm{m}\end{array}$ & 5.77 & 6.27 & 7.30 \\
\hline
\end{tabular}

Figure 18 shows the tool paths generated on a certain cross section using the created workpiece shape and the target shape position and orientation. The tool paths are generated by commercial CAM software (DP Technology, ESPRIT 2016) and Table 2 summarizes the comparison of results of machining efficiency by a commercial machine simulator (Aikoku Alpha G-navi 2.7). As shown in Fig. 18, the outmost tool path length of Case C is shorter than those of the other two cases due to appropriate target shape position and orientation. Moreover, it can be seen that unnecessary tool paths are generated in Case A and B. As a result, the removal volume per tool-path distance decreases and machining time increased compared to Case $\mathrm{C}$. Therefore, the effectiveness of the proposed machine procedure is verified in that the machining efficiency of Case $\mathrm{C}$ is the highest.

In the proposed machining procedure, the acquisition of $2 \mathrm{D}$ images for $\mathrm{SfM}$ including the setup of camera is required. In this case study, the preparation time of these processes is about 30 minutes as the additional time before a machining operation. However, when the differences exist between the 3D model of workpiece and the actual object, various problems may happen such as the occurrence of inappropriate uncut and the interferences between the machine structure and the workpiece. They may require the unnecessary costs as to modify the tool paths and repair the machine tool, and these costs generally become higher. As a result, it is recognized that the proposed machining procedure has a potential to be effective for complicated workpiece shape machining.

\section{Conclusion}

Multifunctional machine tools that allow integration of machining and other manufacturing operations such as AM have been developed and the demand for machining of complicated workpiece shapes has increased. However, it is difficult to predict workpiece shapes created by AM and unexpected collisions can occur between machine structures and a workpiece. Moreover, machining efficiency may decrease due to differences between the assumed workpiece shape and the actual object. Therefore, the aim of this study is to realize highly efficient machining of complicated workpiece shapes corresponding to actual workpiece shapes as created by AM. The proposed machining procedure obtains a 3D model of the workpiece corresponding to the actual object by SfM on a multifunctional machine tool, and the target shape position and orientation are decided using the workpiece shape.

The results of a case study show that a 3D model of a complicated workpiece corresponding to an actual object can be created based on on-machine measurement by SfM, and that adequate target shape position and orientation can be decided so as to shorten the tool path length according to the workpiece shape.

In the proposed machining procedure, the acquisition of 2D images for SfM including the setup of camera is required. However, the proposed machining procedure can avoid the differences between the assumed workpiece shape and the actual object, and the target shape can be located within the workpiece shape. Therefore, the proposed machining procedure does not require to modify the tool paths and ensures collision-free machining between the machine tool structure and workpiece. Additionally, some advanced machine tools that have already installed the camera function, do not need the extra setup of camera and can automate the acquisition of $2 \mathrm{D}$ images. Thus, it is assumed that the cost required for on-machine measurement process decrease in the near future. As a result, it is recognized that the proposed machining procedure has a potential to be effective for complicated workpiece shape machining. 


\section{References}

Abele E. and Korff D., Avoidance of collision-caused spindle damages - challenges, methods and solutions for high dynamic machine tools, CIRP Annals, Vol.60, Issue 1 (2011), pp 425-428.

Arun K. S., Huang T. S. and Blostein S. D., Least-squares fitting of two 3-D point sets, IEEE Transactions on Pattern Analysis and Machine Intelligence, Vol.PAMI-9, Issue 5 (1987), pp.698-700.

Baier W. and Rando C., Developing the use of Structure-from-Motion in mass grave documentation, Forensic Science International, Vol.261 (2016), pp 19-25.

Chen F. J., Yin S. H., Huang H., Ohmori H., Wang Y., Fan Y. F. and Zhu Y. J., Profile error compensation in ultra-precision grinding of aspheric surfaces with on-machine measurement, International Journal of Machine Tools \& Manufacture, Vol.50 (2010), pp.480-486.

Chen L., Xu K. and Tang K., Collision-free tool orientation optimization in five-axis machining of bladed disk, Journal of Computational Design and Engineering, Vol.2 (2015), pp 197-205.

Chen Y. L., Cai Y., Shimizu Y., Ito S., Gao W. and Ju B. F., On-machine measurement of microtool wear and cutting edge chipping by using a diamond edge artifact, Precision Engineering, Vol. 43 (2016), pp.462-467.

Dou J. and Li J., Robust object detection based on deformable part model and improved scale invariant feature transform, Optic, Vol.124 (2013), pp.6485-6492.

Hu P., Tang K. and Lee C. H., Global obstacle avoidance and minimum workpiece setups in five-axis machining, Computer-Aided Design, Vol.45 (2013), pp 1222-1237.

Horn B. K., Closed-form solution of absolute orientation using unit quaternions, Journal of the Optical Society of America A, Vol.4, Issue 4 (1987), pp.629-642.

Horn B. K., Hilden H. M. and Negahdaripour S., Closed-form solution of absolute orientation using orthonormal matrices, Journal of the Optical Society of America A, Vol.5, Issue 7 (1988), pp.1127-1135.

Hossein-Nejad Z. and Nasri M., An adaptive image registration method based on SIFT features and RANSAC transform, Computers and Electrical Engineering, Vol.62 (2017), pp.524-537.

Ibaraki S., Iritani T. and Matsubara T., Error map construction for rotary axes on five-axis machine tools by on-themachine measurement using a touch-trigger probe, International Journal of Machine Tools \& Manufacture, Vol.68 (2013), pp.21-29.

Kaneda.T. and Morishige K., Tool path generation for five-axis controlled machining with consideration of structural interference, Int. J. of Automation Technology, Vol.6, No 6 (2012), pp 710-716.

Lacharnay V., Lavernhe S., Tournier C. and Lartigue C., A physically-based model for global collision avoidance in5axis point milling, Computer-Aided Design, Vol.64 (2015), pp 1-8.

Lowe D. G., Distinctive image features from scale-invariant keypoints, International Journal of Computer Vision, Vol.60, Issue2 (2004), pp.91-110.

Luhmann T., Franser C. and Maas H. G., Sensor modelling and camera calibration for close-range photogrammetry, ISPRS Journal of Photogrammetry and Remote Sensing, Vol.115 (2016), pp.37-46.

Ogawa K., Nakagawa H. and Iwao T., High-Efficiency Machining Strategy for Non-Uniformly Shaped Workpiece Using On-Machine Measurement, International Journal of Automation Technology, Vol.6, No.6 (2012), pp.704-709.

Szipka K., Laspas T. and Archenti A., Measurement and analysis of machine tool errors under quasi-static and loaded conditions, Precision Engineering, Vol. 51 (2018), pp.59-67.

Ukita M. and Takaya Y., On-machine dimensional measurement of large parts by compensating for volumetric errors of machine tools, Precision Engineering, Vol.43 (2016), pp.200-210.

Walker M. W., Shao L. and Volz R. A., Estimating 3-D location parameters using dual number quaternions, CVGIP:Image Understanding, Vol.54, Issue 3 (1991),pp.358-367.

Watanabe K., Kaneko J. and Horio K., Development of tool collision avoidance method adapted to uncut workpiece shape, Int. J. of Automation Technology, Vol.11, No.2 (2017), pp 235-241. 